\title{
Feasibility and analgesic efficacy of the transversus abdominis plane block after single-port laparoscopy in patients having bariatric surgery
}

This article was published in the following Dove Press journal: Journal of Pain Research

26 November 2013

Number of times this article has been viewed

\author{
Michael Wassef \\ David Y Lee \\ Jun L Levine \\ Ronald E Ross \\ Hamza Guend \\ Catherine Vandepitte \\ Admir Hadzic \\ Julio Teixeira
}

Department of Anesthesiology, St Luke's-Roosevelt Hospital Center, New York, NY, USA
Correspondence: Michael Wassef St. Luke's-Roosevelt Hospital Center, Department on Anesthesiology, 5 I5 W 59th St, New York, NY 10019 , USA Email wadsha2y@gmail.com
Purpose: The transversus abdominis plane (TAP) block is a technique increasingly used for analgesia after surgery on the anterior abdominal wall. We undertook this study to determine the feasibility and analgesic efficacy of ultrasound-guided TAP blocks in morbidly obese patients. We describe the dermatomal spread of local anesthetic in TAP blocks administered, and test the hypothesis that TAP blocks decrease visual analog scale (VAS) scores.

Patients and methods: After ethics committee approval and informed consent, 35 patients with body mass index $>35$ undergoing single-port sleeve gastrectomy (SPSG) were enrolled. All patients received balanced general anesthesia, followed by intravenous patient-controlled analgesia (IV-PCA; hydromorphone) postoperatively; all reported VAS $>3$ upon arrival to the recovery room. From the cohort of 35 patients having single-port laparoscopy (SPL), a sealed envelope method was used to randomly select ten patients to the TAP group and 25 patients to the control group. The ten patients in the TAP group received ultrasound-guided TAP blocks with $30 \mathrm{~mL}$ of $0.2 \%$ Ropivacaine injected bilaterally. The dermatomal distribution of the sensory block (by pinprick test) was recorded. VAS scores for the first 24 hours after surgery and opioid use were compared between the IV-PCA+TAP block and IV-PCA only groups.

Results: Sensory block ranged from T5-L1. Mean VAS pain scores decreased from $8 \pm 2$ to $4 \pm 3(P=0.04)$ within 30 minutes of TAP block administration. Compared with patients given IV-PCA only, significantly fewer patients who received TAP block had moderate or severe pain (VAS 4-10) after block administration at 6 hours and 12 hours post-surgery. However, cumulative consumption of hydromorphone at 24 hours after SPSG surgery was similar for both groups. Conclusion: Ultrasound-guided TAP blocks in morbidly obese patients are feasible and result in satisfactory analgesia following SPSG in the immediate postoperative period.

Keywords: regional anesthesia, postoperative pain, ultrasound, analgesia, nerve blocks

\section{Introduction}

The transversus abdominis plane (TAP) block is a relatively new regional anesthetic technique that targets the sensory nerve supply of the anterior-lateral abdominal wall. First described by Rafi et al in 2001, the block is performed by injecting local anesthetic into the plane between the internal oblique and the transversus abdominis muscles using the triangle of Petit as a landmark. ${ }^{1}$ This TAP plane is infiltrated with local anesthetics to target the T7-T12 intercostal nerves, the ilioinguinal, iliohypogastric, and the lateral cutaneous branches of the dorsal rami of L1-L3. ${ }^{2,3}$ TAP blocks have been implemented successfully for pain control after laparoscopic surgery in nonobese patients undergoing diverse procedures ranging from appendectomy to neurostimulator implants. ${ }^{4-11}$ The resulting analgesia may be especially beneficial in 
morbidly obese patients after abdominal surgery due to their higher risk for postoperative pulmonary complication. ${ }^{12,13}$ The introduction of ultrasound guidance has allowed greater precision of needle placement in the desired tissue plane. ${ }^{14}$ While several modifications of this technique have been described, a universally applicable technique has not been agreed upon by all authors. ${ }^{2,14}$

Single-port laparoscopy (SPL) along with natural orifice transluminal endoscopic surgery and endoluminal operations are becoming common. SPL utilizes a single surgical incision in a central abdominal location that may be suitable for accomplishing analgesia with TAP blocks. However, visualization of the abdominal wall muscles can be hindered by morbid obesity and could lead to failed regional anesthesia. ${ }^{15}$ We undertook this study to determine the feasibility of using ultrasound-guided TAP blocks in patients with morbid obesity undergoing SPL. We describe the dermatomal spread of ultrasound-guided TAP blocks in morbidly obese patients, and test the hypothesis that TAP blocks decrease pain following single-port sleeve gastrectomy (SPSG). Recently, a report by Sinha et al, showed a benefit of TAP blocks in the morbidly obese undergoing multiport laparoscopy. ${ }^{16}$ This study, however, aims at discerning the benefits of TAP in addressing the pain created by the deep incision, due to the single port, which we felt it was uniquely suitable to address.

\section{Methods}

This study was approved by the Institutional Review Board of St Luke's-Roosevelt Hospital Center. A total of 35 patients undergoing SPSG were enrolled. Inclusion criteria were ASA (American Society of Anesthesiologists) physical status 2-3, body mass index $(\mathrm{BMI})>35$, and visual analog scale (VAS) $>3$ upon arrival to the post-anesthesia care unit (PACU) despite intravenous patient-controlled analgesia (IV-PCA). In the cohort of 35 patients having SPL, a sealed envelope method was used to randomly select ten patients to the TAP group and 25 patients to the control group. All patients received standardized balanced general anesthesia. Premedication consisted of midazolam $2 \mathrm{mg} I V$; induction with propofol (2 mg/kg), fentanyl ( $2 \mu \mathrm{g} / \mathrm{kg})$, rocuronium (0.6 mg/ $\mathrm{kg}$ ), and maintenance with desflurane $6 \%-8 \%$. Local infiltration of the surgical site was not performed by the surgeon so as not to present a confounding variable. SPL was performed using the SIL ${ }^{\circledR}$ port (Covidien Ltd., Mansfield, MA, USA), placed transumbilically through a $2 \mathrm{~cm}$ incision.

Following completion of the surgical procedure, the patients were allowed to wake up, extubated and transported to the PACU. All patients received PCA with IV hydromorphone (loading dose 1-2 $\mathrm{mg}$ and demand dose of $0.2 \mathrm{mg}$ every 8 minutes for a maximum dose of $6 \mathrm{mg}$ in a 4-hour period). From the cohort of 35 patients, ten were randomly selected to receive ultrasound-guided TAP blocks upon arrival to the PACU by the, aforementioned, sealed envelope method. The TAP block was performed bilaterally by injecting $30 \mathrm{~mL}$ of $0.2 \%$ Ropivacaine at the mid-axillary line at the level of the rib cage. Due to the depth of the abdominal wall structures, a low frequency curved transducer (SonoSite C60x/5-2 MHz 2010-06; SonoSite Inc., Bothel, WA, USA) was used to guide needle placement. Layers of the abdominal wall (internal oblique, external oblique, and transversus abdominis) were identified, and a $22 \mathrm{G}, 10 \mathrm{~cm}$ long needle (BD, Franklin Lakes, NJ, USA) was inserted into the TAP plane to inject local anesthetic under direct visualization.

Upon block placement, the reports of pain on the VAS scale (ranging from 0 indicating no pain to 10 indicating extreme pain) and requirement for opioids was assessed every 5 minutes for the first 30 minutes, then hourly for the next 12 hours and every 6 hours thereafter until 24 hours after surgery. Whenever pain occurred during needle advancement, we injected $1 \mathrm{~mL}$ of local anesthetic before continuing to advance the needle. While we did not specifically study the discomfort during the procedure, our observation was that it was not very uncomfortable for the patients.

Patients who received IV-PCA+TAP block $(n=10)$ and those who received IV-PCA only $(n=25)$ were compared for opioid consumption, pain score, and any complications such as hematoma at the site of injection and local anesthetic toxicity. Also, a pinprick test 30 minutes after block placement determined the extent of the sensory blockade accomplished with the TAP block. Evaluation of the pinprick test and the VAS scores were performed by a research assistant blinded to the intervention and the purpose of this study.

Sample size for this study was estimated for $\alpha=0.05$, power $=0.90$, 4-point difference in VAS (standard deviation 3), and a 10:25 case/control ratio. Ten patients were required for the IV-PCA+TAP block group, and 25 patients were assigned to the IV-PCA-only group. Differences between the groups for continuous variables (age, BMI, total PCA) were tested by the Student's $t$-test; however, pre-post difference in pain scores for the TAP group was compared by the paired $t$-test. Differences between the groups on categorical data (sex) were tested by Pearson $X^{2}$ or Fisher's exact test, as appropriate. Postoperative pain scores were categorized as no pain (VAS 0), mild (VAS 1-3), moderate (VAS 4-7), or severe (VAS 8-10), and compared between the groups by the Kruskal-Wallis $H$ test. Differences were 
considered statistically significant at $P<0.05$. All analyses were conducted with SPSS (SPSS Inc., Chicago, IL, USA) software, version 16.

\section{Results}

A total of 35 patients were studied; ten patients received IV-PCA+TAP block, and 25 patients received IV-PCA only. Age and BMI did not differ between the two groups (Table 1). There were slightly more females among the patients who had IV-PCA only than among those who received IV-PCA+TAP block, but this difference was not statistically significant (2-sided Fisher's exact $P=0.17$ ). The TAP block in bariatric patients resulted in a distribution of sensory block ranging from T5-L1 (Figure 1). All patients who received the TAP block reported absence of sensation in the periumbilical region, as assessed by pinprick test.

The VAS scores were categorized into four categories to better describe the intensity of pain as: no pain (0), mild (1-3), moderate (4-7), and severe (8-10). VAS scores decreased among patients who received the TAP block from

Table I Demographic and clinical characteristics and postoperative pain between IV-PCA+TAP block and IV-PCA-only groups

\begin{tabular}{|c|c|c|c|}
\hline & $\begin{array}{l}\text { IV-PCA+TAP } \\
\text { block }(n=10)\end{array}$ & $\begin{array}{l}\text { IV-PCA } \\
\text { only }(n=25)\end{array}$ & $P$-value \\
\hline Age $(y)$ & $43 \pm 12$ & $47 \pm 13$ & ns \\
\hline \multicolumn{4}{|l|}{ Sex } \\
\hline Male & $4(40)$ & $3(12)$ & ns \\
\hline Female & $6(60)$ & $22(88)$ & \\
\hline BMI $\left(\mathrm{kg} / \mathrm{m}^{2}\right)$ & $42 \pm 4$ & $47 \pm 7$ & ns \\
\hline \multicolumn{4}{|l|}{ Postoperative pain (VAS) } \\
\hline \multicolumn{4}{|l|}{6 hours } \\
\hline No pain (0) & 9 & 12 & 0.04 \\
\hline Mild (I-3) & 1 & 9 & \\
\hline Moderate (4-7) & 0 & 3 & \\
\hline Severe $(8-10)$ & 0 & I & \\
\hline \multicolumn{4}{|l|}{12 hours } \\
\hline No pain $(0)$ & 9 & 12 & 0.05 \\
\hline Mild $(0-I)$ & 1 & 10 & \\
\hline Moderate (2-5) & 0 & 3 & \\
\hline Severe $(6-10)$ & 0 & 0 & \\
\hline \multicolumn{4}{|l|}{24 hours } \\
\hline No pain (0) & 9 & 17 & ns \\
\hline Mild $(0-I)$ & 0 & 8 & \\
\hline Moderate (2-5) & 1 & 0 & \\
\hline Severe $(6-10)$ & 0 & 0 & \\
\hline $\begin{array}{l}\text { IV-PCA 24-hour } \\
\text { consumption } \\
\text { (mg hydromorphone) }\end{array}$ & $4 \pm 2$ & $6 \pm 3$ & ns \\
\hline
\end{tabular}

Note: Data are mean \pm standard deviation for continuous variables; $n$ (\%) for categorical variables.

Abbreviations: IV-PCA, intravenous patient-controlled analgesia; TAP, transversus abdominis plane; BMI, body mass index; VAS, visual analog scale; ns, not significant; n, number; $y$, year.
$8 \pm 2$ to $4 \pm 3(P=0.04)$ within 30 minutes of TAP block administration. Level of pain was lower in the IV-PCA+TAP block group compared with the IV-PCA-only group at 6 hours and 12 hours, but the groups did not differ by 24 hours (Table 1). There was no statistically significant difference between the groups in cumulative opioid consumption at 24 hours (Table 1). No complications were related to the administration of the TAP block.

\section{Discussion}

Ultrasound-guided TAP blocks in morbidly obese patients undergoing SPSG resulted in a consistent sensory block and lower VAS scores within 30 minutes of injection. Patients with IV-PCA+TAP block also had lower VAS scores at 6 hours and 12 hours post-surgery. However, VAS scores and cumulative opioid consumption at 24 hours were similar between the IV-PCA+TAP block and IV-PCA only group.

The TAP block has been previously studied in patients having open and laparoscopic surgical procedures such as Cesarean delivery, hysterectomy, inguinal and ventral abdominal hernia repair, cholecystectomy, appendectomy, bowel resection, retropubic prostatectomy, neurostimulator implantation, and bone grafting. ${ }^{4-11}$ Most studies have demonstrated that the TAP block decreases peri- and postoperative pain and reduces the use of opioids..$^{5,17-21}$ Recent studies have shown similar benefits in obese patients undergoing laparoscopic colorectal surgery and Cesarean delivery. ${ }^{22,23}$ Decreasing the pain in the immediate postoperative period is of substantial importance for perioperative management of morbidly obese patients, as it may decrease the need for opioids at the critical times of emergence from general anesthetic, extubation, and immediate management in the PACU. ${ }^{12,13}$ While epidural analgesia is a feasible analgesic modality, the technical challenge of placing an epidural catheter in this patient population often precludes its use in everyday clinical practice. ${ }^{24}$ Although our study was not powered for safety, we did not record any complications. When TAP block was first described, obesity was thought to be one of the contraindications to performing the block because it is difficult to identify the triangle of Petit in obese patients. ${ }^{25,26}$ However, ultrasound guidance allowed identification of the layers of the abdominal wall even in obese patients (average BMI 42 in our study population) where landmarks are often obscured by the body habitus. ${ }^{16}$

In conclusion, ultrasound-guided TAP block is a feasible and effective technique for postoperative analgesia in morbidly obese patients having SPSG. Future studies are indicated to determine whether the relatively short-lived 


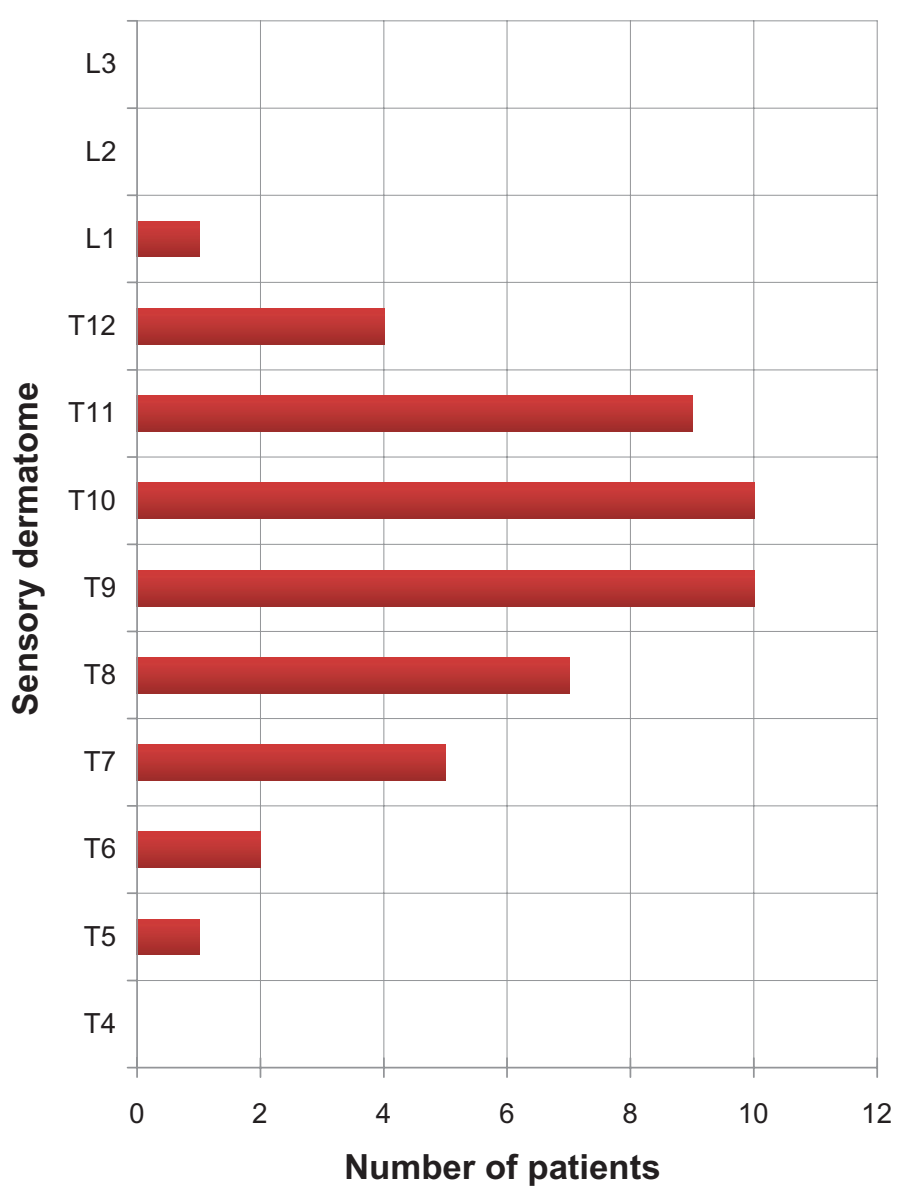

Number of patients reporting numbness at specific dermatomes

Figure I Dermatomal sensory distribution of the transversus abdominis plane block.

analgesic benefit in patients having SPSG can be extended with catheter infusion, longer-acting local anesthetic formulations (eg, encapsulated bupivacaine), or other techniques for a more sustained analgesic benefit.

\section{Disclosure}

The authors report no conflicts of interest in this study.

\section{References}

1. Rafi AN. Abdominal field block: a new approach via the lumbar triangle. Anaesthesia. 2001;56(10):1024-1026.

2. McDonnell JG, O'Donnell BD, Farrell T, et al. Transversus abdominis plane block: a cadaveric and radiological evaluation. Reg Anesth Pain Med. 2007;32(5):399-404.

3. El-Dawlatly AA, Turkistani A, Kettner SC, et al. Ultrasound-guided transversus abdominis plane block: description of a new technique and comparison with conventional systemic analgesia during laparoscopic cholecystectomy. Br J Anaesth. 2009;102(6):763-767.

4. O'Donnell BD, McDonnell JG, McShane AJ. The transversus abdominis plane (TAP) block in open retropubic prostatectomy. Reg Anesth Pain Med. 2006;31(1):91.

5. Baaj JM, Alsatli RA, Majaj HA, Babay ZA, Thallaj AK. Efficacy of ultrasound-guided transversus abdominis plane (TAP) block for postcesarean section delivery analgesia - a double-blind, placebo-controlled, randomized study. Middle East J Anesthesiol. 2010;20(6):821-826.

6. Allcock E, Spencer E, Frazer R, Applegate G, Buckenmaier C 3rd. Continuous transversus abdominis plane (TAP) block catheters in a combat surgical environment. Pain Med. 2010;11(9):1426-1429.
7. McMorrow RC, Ni Mhuircheartaigh RJ, Ahmed KA, et al. Comparison of transversus abdominis plane block vs spinal morphine for pain relief after Caesarean section. Br J Anaesth. 2011;106(5): 706-712.

8. Chiono J, Bernard N, Bringuier S, et al. The ultrasound-guided transversus abdominis plane block for anterior iliac crest bone graft postoperative pain relief: a prospective descriptive study. Reg Anesth Pain Med. 2010;35(6):520-524.

9. Tanaka M, Mori N, Murakami W, et al. [The effect of transversus abdominis plane block for pediatric patients receiving bone graft to the alveolar cleft.] Masui. 2010;59(9):1185-1189. Japanese.

10. Asensio-Samper JM, De Andres-Ibanez J, Fabregat Cid G, Villanueva Perez V, Alarcon L. Ultrasound-guided transversus abdominis plane block for spinal infusion and neurostimulation implantation in two patients with chronic pain. Pain Pract. 2010;10(2):158-162.

11. McDonnell JG, Curley G, Carney J, et al. The analgesic efficacy of transversus abdominis plane block after cesarean delivery: a randomized controlled trial. Anesth Analg. 2008;106(1):186-191.

12. Ahmad S, Nagle A, McCarthy RJ, Fitzgerald PC, Sullivan JT, Prystowsky J. Postoperative hypoxemia in morbidly obese patients with and without obstructive sleep apnea undergoing laparoscopic bariatric surgery. Anesth Analg. 2008;107(1):138-143.

13. Rosenberg-Adamsen S, Lie C, Bernhard A, Kehlet H, Rosenberg J. Effect of oxygen treatment on heart rate after abdominal surgery. Anaesthesiology. 1999;90(2):380-384.

14. Hadzic A. Textbook of Regional Anesthesia and Acute Pain Management. 1st ed. New York: McGraw-Hill; 2007.

15. Nielsen KC, Guller U, Steele SM, Klein SM, Greengrass RA, Pietrobon R. Influence of obesity on surgical regional anesthesia in the ambulatory setting: an analysis of 9,038 blocks. Anaesthesiology. 2005;102(1):181-187. 
16. Sinha A, Jayaraman L, Punhani D. Efficacy of ultrasound-guided transversus abdominis plane block after laparoscopic bariatric surgery: a double blind, randomized, controlled study. Obes Surg. 2013;23(4): 548-553.

17. Siddiqui MR, Sajid MS, Uncles DR, Cheek L, Baig MK. A metaanalysis on the clinical effectiveness of transversus abdominis plane block. J Clin Anesth. 2011;23(1):7-14.

18. Erol DD, Yilmaz S, Polat C, Arikan Y. Efficacy of thoracic epidural analgesia for laparoscopic cholecystectomy. Adv Ther. 2008;25(1): 45-52.

19. van Zundert AA, Stultiens G, Jakimowicz JJ, van den Borne BE, van der Ham WG, Wildsmith JA. Segmental spinal anaesthesia for cholecystectomy in a patient with severe lung disease. Br J Anaesth. 2006;96(4):464-466.

20. van Zundert AA, Stultiens G, Jakimowicz JJ, et al. Laparoscopic cholecystectomy under segmental thoracic spinal anaesthesia: a feasibility study. Br J Anaesth. 2007;98(5):682-686.

21. Wyniecki A, Zetlaoui P, Bruyere M, Benhamou D. [Bilateral catheter for continuous TAP block and postoperative pain relief after gynecologic surgery.] Ann Fr Anesth Reanim. 2011;30(1):67-69. French.
22. Singh S, Dhir S, Marmai K, Rehou S, Silva M, Bradbury C. Efficacy of ultrasound-guided transversus abdominis plane blocks for post-cesarean delivery analgesia: a double-blind, dose-comparison, placebo-controlled randomized trial. Int J Obstet Anesth. 2013;22(3):188-193.

23. Walter CJ, Maxwell-Armstrong C, Pinkney TD, et al. A randomised controlled trial of the efficacy of ultrasound-guided transversus abdominis plane (TAP) block in laparoscopic colorectal surgery. Surg Endosc. 2013;27(7):2366-2372.

24. Rawal N. Epidural technique for postoperative pain: gold standard no more? Reg Anesth Pain Med. 2012;37(3):310-317.

25. Kishore K, Agarwal A. Ultrasound-guided continuous transverse abdominis plane block for abdominal surgery. J Anaesth Clin Pharmacol. 2011;27(3):336-338.

26. McDonnell JG, Laffey JG. The transversus abdominis plane block. Anesth Analg. 2007;105(3):883.
Journal of Pain Research

\section{Publish your work in this journal}

The Journal of Pain Research is an international, peer-reviewed, open access, online journal that welcomes laboratory and clinical findings in the fields of pain research and the prevention and management of pain. Original research, reviews, symposium reports, hypothesis formation and commentaries are all considered for publication.

\section{Dovepress}

The manuscript management system is completely online and includes a very quick and fair peer-review system, which is all easy to use. Visit http://www.dovepress.com/testimonials.php to read real quotes from published authors. 